\title{
Optical Coherence Tomography in Glaucoma
}

\author{
P Sathyan, Shilpa, Anitha \\ Glaucoma Services, Aravind Eye Hospital, Coimbatore, Tamil Nadu, India
}

Correspondence: P Sathyan, Glaucoma Services, Aravind Eye Hospital, Avinashi Road, Coimbatore-641014, Tamil Nadu, India e-mail: sathyan.p@cbe.aravind.org,dr.sathyan.p@gmail.com

\section{ABSTRACT}

The optic disk and the RNFL are the principal sites of apparent glaucomatous damage which precedes glaucomatous visual field alterations. RNFL defects are known to precede detection of visual field defects by approximately 6 years. Accurate early detection and monitoring of ONH and RNFL defects has become the prime focus of effective management of glaucoma. Optical coherence tomography employs low-coherence interferometry to obtain cross-sectional images of the ocular tissues.

This review attempts to critically analyse the applications of both, anterior and posterior segment OCT in glaucoma management.

Keywords: AS-OCT, RNFL OCT, Glaucoma diagnosis.

Glaucoma is an optic neuropathy with characteristic optic nerve appearance and visual field loss for which elevated intraocular pressure (IOP) is one of the main risk factors. ${ }^{1}$ This characteristic optic nerve appearance results from structural glaucomatous changes which usually precede functional deterioration (visual field loss). Worldwide, glaucoma is the most common cause of irreversible blindness and the second leading cause of blindness. ${ }^{2}$ The optic disk and the RNFL are the principal sites of apparent glaucomatous damage which precede glaucomatous visual field alterations. In $60 \%$ of reported cases, retinal nerve fiber layer (RNFL) defects preceded detection of visual field defects by approximately 6 years. ${ }^{3}$ Accurate early detection and monitoring of optic nerve head (ONH) and RNFL defects have become the prime focus of effective management of glaucoma.

\section{INTRODUCTION}

Optical coherence tomography (OCT) is an imaging technology that employs low-coherence interferometry to obtain crosssectional images of the ocular tissues. The principle of OCT is analogous to that of ultrasonography, but uses light instead of sound to acquire high resolution images of the ocular structures. A beam of light is shone on the eye and reflections returning from the structures are analyzed to produce real time images.

\section{PRINCIPLES OF OCT}

A light source is directed to a partially reflecting mirror that splits the light into two beams: One is directed toward a mirror placed at a known distance (reference mirror) and the other is directed toward the eye, from where it will reflect back. The back-reflected light from the eye is combined with the backreflected light from the reference mirror and coherent light is compared. Interference is produced when two light pulses coincide. The reference mirror is then moved so that the time delay of the reference light pulse can change accordingly and therefore other intraocular structures can be measured. The laser beam is passed throughout the tissue and a series of scans are obtained to produce a two-dimensional map.

\section{APPLICATION OF OCT IN GLAUCOMA}

\section{Anterior Segment-OCT}

Anterior segment OCT (AS-OCT) uses light of longer wavelength $(1310 \mathrm{~nm})$ to obtain images of the anterior segment. Light-induced damage of the retina at this high wavelength is prevented by the absorption of light by the water in the ocular tissues.

\section{Applications of AS-OCT}

1. Evaluation of angle structures. It causes minimal distortion of the angle anatomy because no visible light is shone on the eye and there is no pressure on the globe.

2. AS-OCT provides detailed calculations of parameters, such as angle opening distance, angle recess area, and the trabecular-iris space area, thus introducing new levels of precision for approaching PAC disease (Fig. 1).

3. To evaluate the effect of laser peripheral iridotomy and other interventions on the angle anatomy, which helps the clinician plan further interventions if necessary.

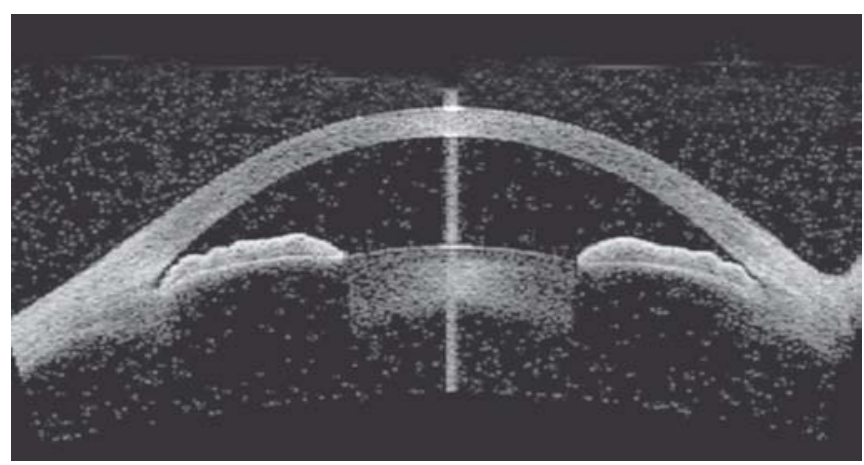

Fig. 1: AS-OCT showing $180^{\circ}$ narrow angles 
4. Assessment of bleb morphology and patency of ostium post-filtering surgery helps the clinician to plan further interventions as necessary.

\section{Posterior Segment-OCT}

Posterior segment OCT uses light of $830 \mathrm{~nm}$ to obtain images of the posterior segment structures, such as $\mathrm{ONH}$, retinal nerve fiber layer and macula. It has software that facilitates image acquisition, storage, retrieval and analysis. Several topographic ONH parameters are automatically calculated and are reported along with a color-coded map and ONH topographic map.

OCT has evolved through different modifications from OCT1 to fourier domain/spectral domain OCT. The newer method of spectral domain (SD) OCT has higher speed and resolution than its predecessor, Stratus OCT (Figs 2 and 3). Spectral-domain OCT also captures three-dimensional images of optic disk and surrounding tissue components. Three of the commonly used SD-OCT devices are the Spectralis (Heidelberg Engineering, Dossenheim, Germany), the Cirrus (Carl Zeiss Meditec, Dublin, CA) and the RTVue (Optovue Inc, Fremont, CA).

Axial resolution of spectral-domain (SD) OCT is twice higher (5-7 microns) than stratus OCT (approximately 10 microns). The SD OCT instruments can acquire B-scans 45 to 130 times faster than Stratus OCT and multiple B-scans can be acquired at the same location, when averaged results in a speckle-noisereduced image with clearly distinguishable boundaries between retinal layers. It also has three-dimensional (3-D) eye tracking system (Spectralis HRA_OCT; Heidelberg Engineering, Heidelberg, Germany) that reduces motion induced artifacts.

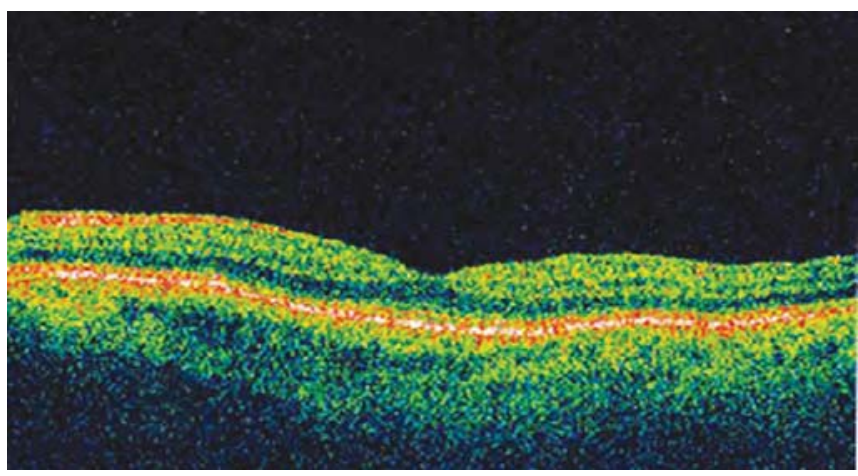

Fig. 2: Stratus OCT

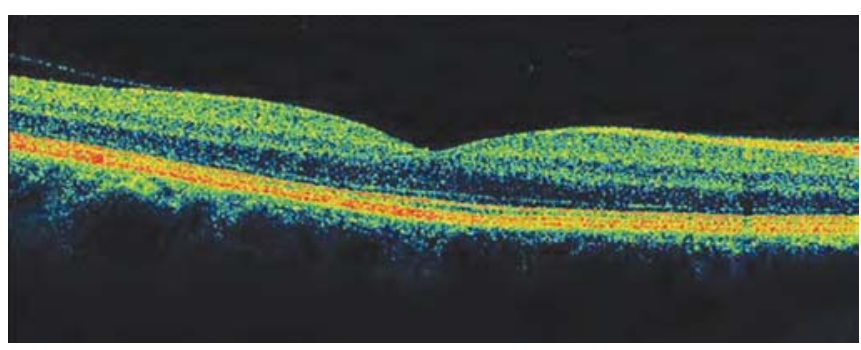

Fig. 3: Spectral domain OCT

\section{Interpretation of OCT (FIGS 4 to 8 )}

\section{Quality Assessment}

1. Appropriate centration of the peripapillary circular scan is essential for accurate measurements of RNFL thickness.

2. Signal strength value of the scan should be greater than 5 .

3. Homogeneity of the RNFL scan is important since loss of reflectivity can affect the overall quality.

The figure shows normative data of Stop-Light color scheme scan with areas of defect in any given patient (normative population). This helps the physician to easily read the chart without much difficulty.

\section{Macular Nerve Fiber Layer Assessment}

OCT scans of the macula involve a strategy of six intersecting lines that intersect at the foveal center. Assessment of the macular region in glaucoma is necessary because over $50 \%$ of retinal ganglion cells lie in the macular region and hence it is the ideal region to detect early cell loss.

In macular thickness analysis by Stratus OCT central $3 \mathrm{~mm}$ of the posterior pole have the highest scan density compared with the outer (3 to $6 \mathrm{~mm}$ ) concentric ring.

The ganglion cell complex (GCC) which includes: (1) the retinal nerve fiber layer (RNFL), (2) the ganglion cell layer (GCL) and (3) the inner-plexiform layer (IPL) becomes thinner due to ganglion cell loss in glaucoma. Spectral OCT (RTVue) measures the thickness of GCC in macular region and gives analysis compared to an extensive normative database.

GCC scan data is displayed as thickness map of GCC layer. This map is color-coded where thicker regions are displayed in hot colors (yellow and orange) and thinner regions in cooler colors (blue and green). GCC map for a normal eye shows a bright circular band surrounding the macula representing thick GCC as depicted below.

\section{Clinical Applications of OCT}

1. Retinal nerve fiber layer analysis: RNFL thickness measurement is graphed in a TSNIT orientation and compared to age matched normative data. Decreased RNFL thickness represents glaucoma.

2. ONH analysis: Disk margins are objectively identified by using signal from and of RPE. Key parameters include cup to disk ratio and horizontal integrated rim volume.

3. Macular thickness analysis: Thinning of macula may reflect glaucomatous loss.

4. A recent software upgrade of Stratus OCT (Stratus OCT Version 5.0) has included the glaucoma progression analysis to evaluate the association between average RNFL thickness and age.

\section{Advantages of OCT}

1. Easy to operate.

2. It has the best resolution among all the imaging devices.

3. Has a rapid image acquistion time. 


\section{SCANNING PROTOCOLS}

Macular scan

Six $6 \mathrm{~mm}$

radial line

scans

RNFL scan

Optic nerve head scan
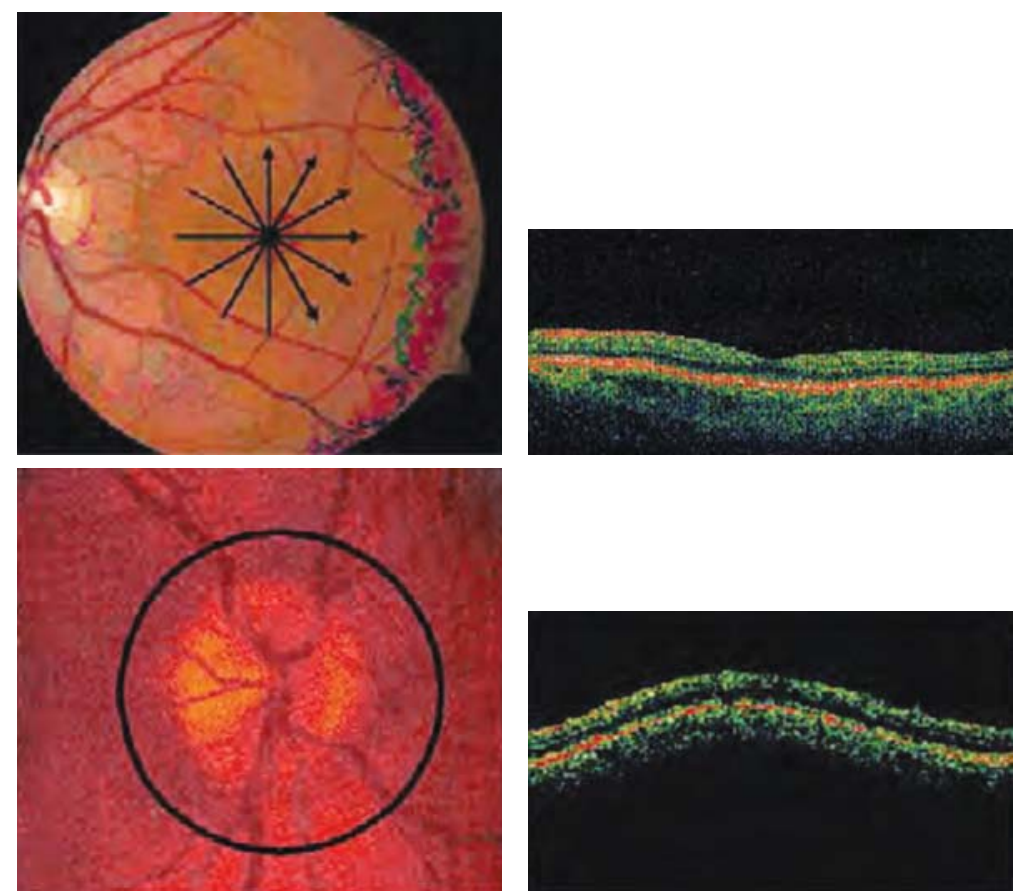

Three $1.73 \mathrm{~mm}$

radius circle scans
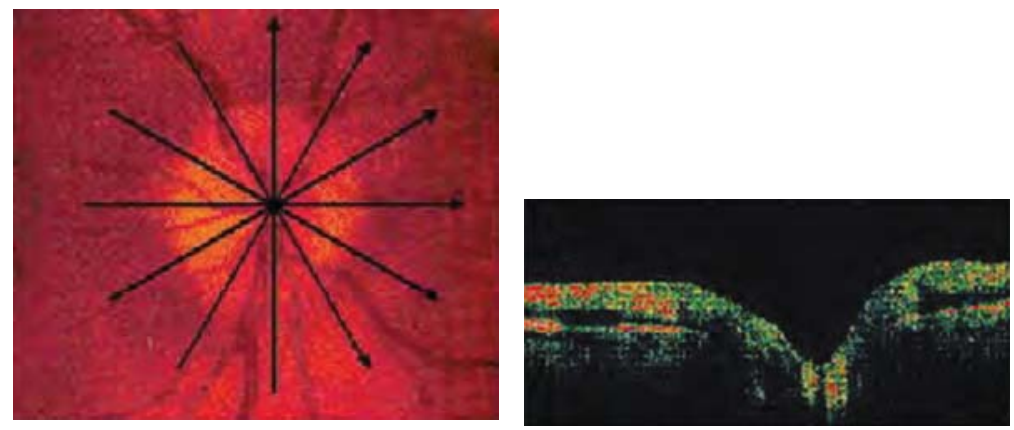

Fig. 4: Scanning protocols

RNFL normative data display

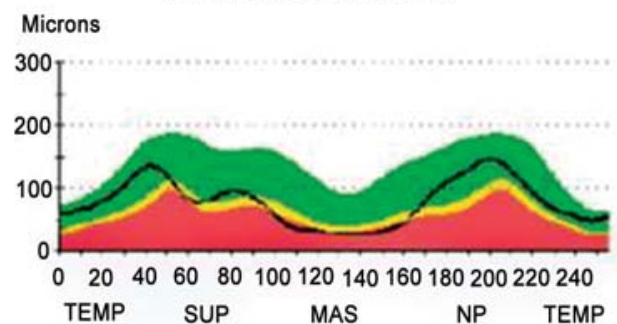

Macula normative data display (thickness chart) Microns

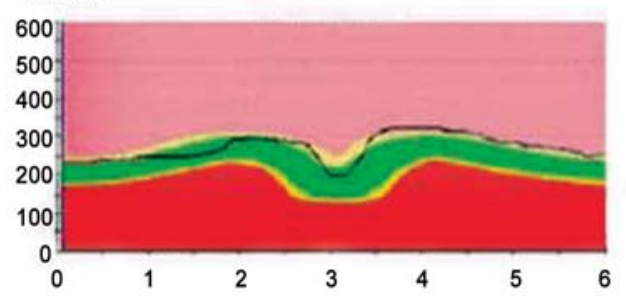

RNFL normative distribution of the normal population

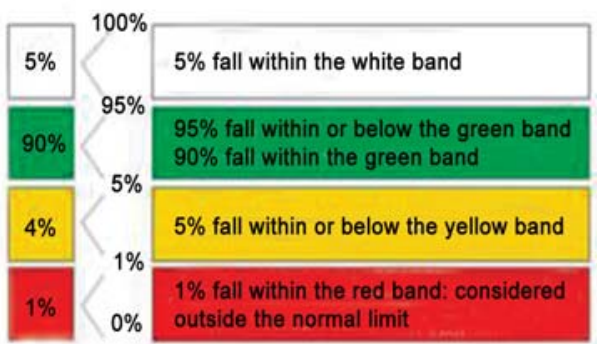

Macula normative distribution of the normal population

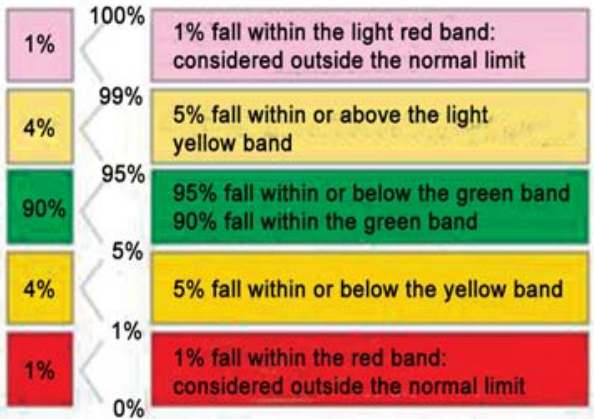

Fig. 5: Statistical significance and normative database 
Patient information

RNFL thickness chart

Peripapillary RNFL thickness is displayed in ISNIT format. Colored bands demonstrate range of normative data.

Sector averages

Comparison to normative data in each sector is indicated with stoplight color scheme. Values are displayed numerically

Quadrant averages

Comparison to normative data in each quadrant is indicated with stoplight color scheme. Values are displayed numerically

OD/OS graph

TSNIT line graph displays RNFL thickness in both eyes.

Asymmetry may be indicative of glaucornatous loss.
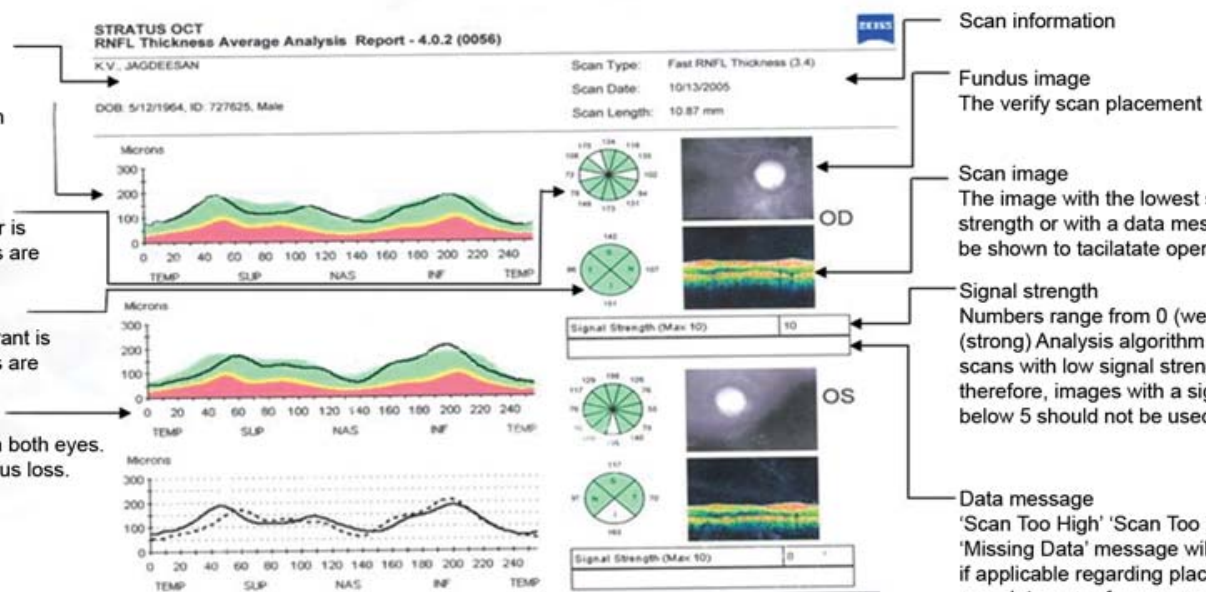

The image with the lowest signal

strength or with a data message will

be shown to tacilatate operator review

Signal strength

Numbers range from 0 (weak) to 10 (strong) Analysis algorithm may fail on scans with low signal strength,

therefore, images with a signal strength below 5 should not be used for analysis

Scans used

If any of the 3 circular scans has been deselected, analysis might be incomplete and for normative data will not be displayed

Lgend

Normative data is displayed in stoplight

colorcode, described in detail in inside back cover

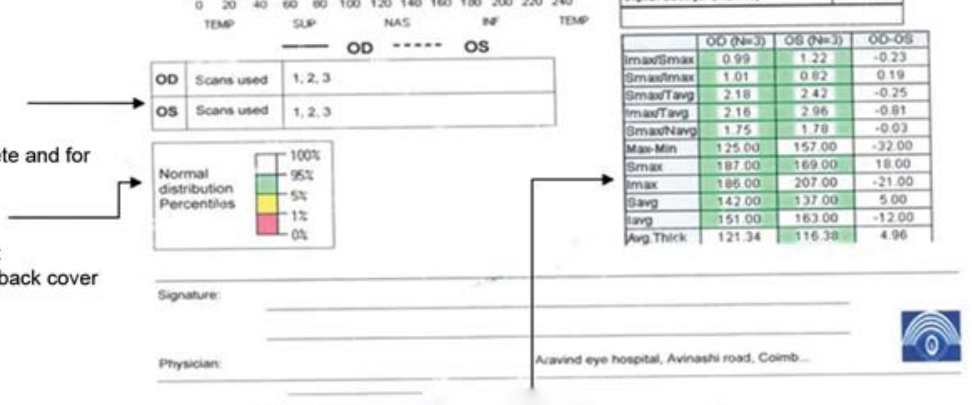

Data message

Scan Too High' 'Scan Too Low' or

'Missing Data' message will be shown if applicable regarding placement and completeness of scan

Tabular data

For each eye, values are compared to normative data and displayed in stoplight color code, described in detail on inside back cover

Fig. 6: RNFL thickness chart

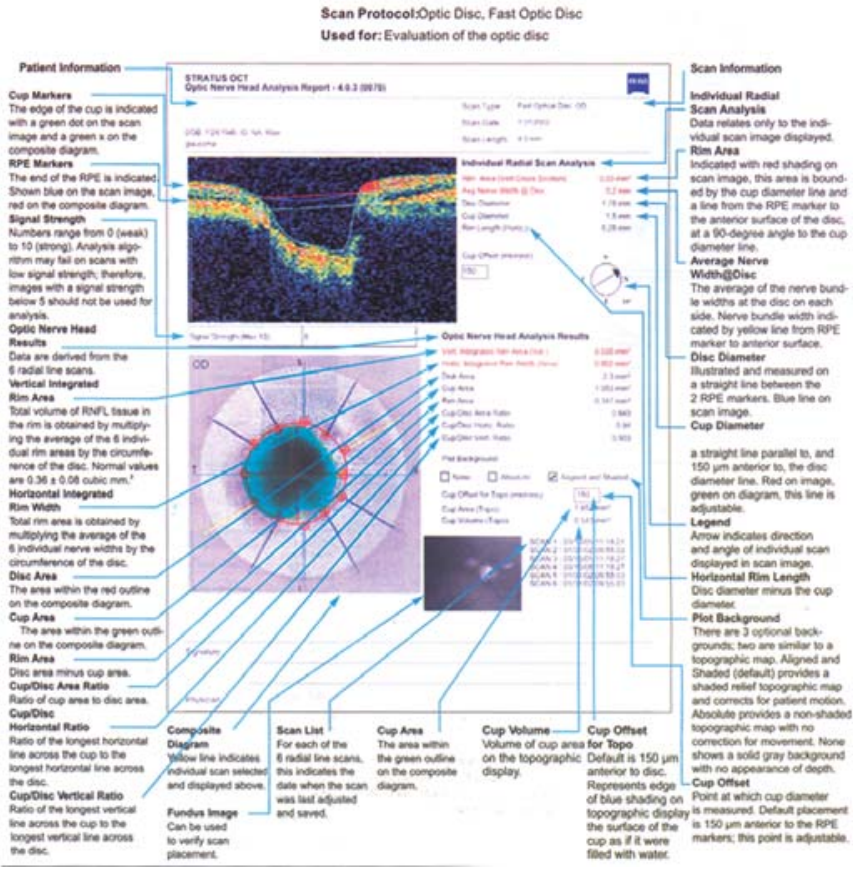

Fig. 7: Optic nerve head analysis chart

4. Being a noncontact technique, images can be obtained without causing undue discomfort to the patient.

5. Qualitative and quantitative data can be collected and analyzed in an objective and reproducible way.

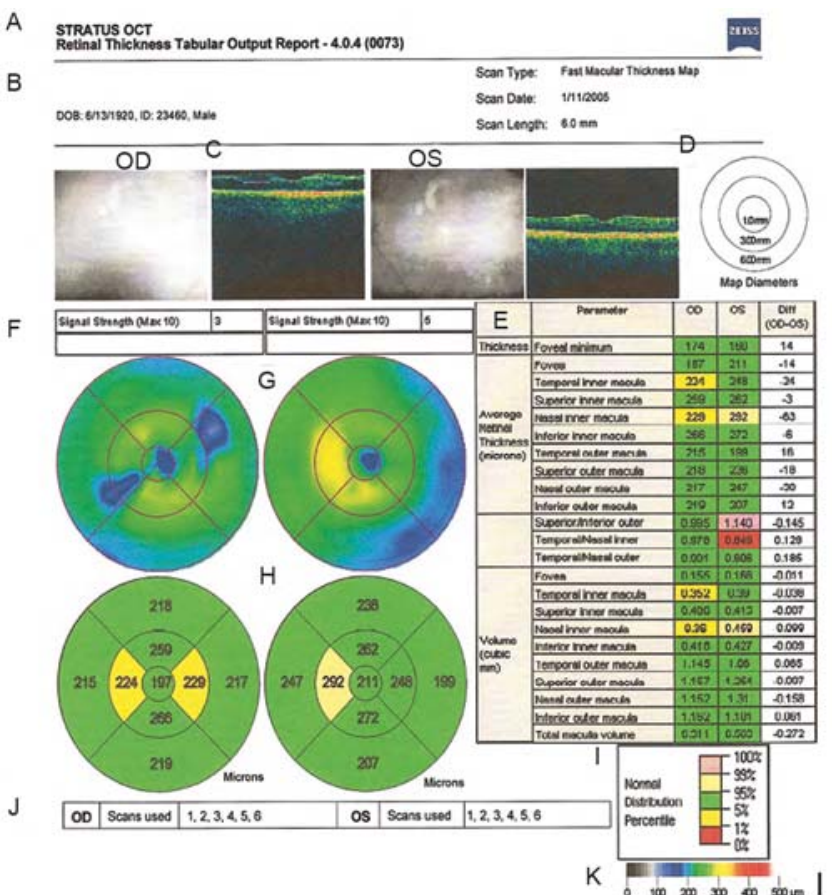

Fig. 8: Macular thickness analysis

6. It is the only technology capable of imaging the optic nerve head, retinal nerve fiber layer and macula.

7. Can obtain posterior segment images without pupillary dilatation. 


\section{Precision of OCT in Early Diagnosis of Glaucoma}

Various studies on $\mathrm{OCT}^{4-8}$ have shown:

- Measurement of RNFL thickness with OCT has been reliable in discriminating normal from glaucomatous eyes.

- OCT has good sensitivity and specificity for differentiating normal from glaucomatous eyes. A study by Chang et al showed Stratus OCT sensitivity and specificity for average RNFL abnormal at the $5 \%$ level were 80 and $94 \%$ respectively and at $1 \%$ level were 61 and $100 \%$ respectively. Cirrus OCT sensitivity and specificity for average RNFL abnormal at the $5 \%$ level were 83 and $88 \%$ respectively and at the $1 \%$ level were 65 and $100 \%$ respectively. ${ }^{9}$

\section{Limitations of OCT}

1. AS-OCT has a poor ability to show the details of ciliary body and the posterior surface of the iris ( since the posterior pigment epithelium of the iris and the ciliary epithelium block the passage of infrared light). Therefore AS-OCT can not detect cyclodialysis clefts and ciliary body tumors.

2. Image quality of superior and inferior quadrants of the angle with AS-OCT is suboptimal due to interference from the eyelids.

3. Landmarks, such as scleral spur and Schwalbe's line are not always clearly visible as with UBM, so quantitative measurements of angle width may not be very accurate.

4. Automatic demarcation of the optic disk borders by the machine may be inaccurate in cases of parapapillary atrophy, which would confound the interpretation of optic disk topography. This may limit the ability of OCT to detect the progression of glaucomatous optic disk damage.

5. It is possible that localized NRR/optic cup changes would be missed by the interpolation algorithm.

6. Depends on the skill of the operator.

7. Poor quality of images in dense media opacities.

8. Difficult in uncooperative patients.

9. Expensive instrumentation.

\section{CONCLUSION}

Medicine and technology are advancing hand in hand to provide quality health care. Technology innovation and improvement will continue to impact health services.
OCT is a new technology. Any new technology introduces both difficulties and opportunities. The lack of large scale normative database is perhaps the greatest issue in interpretation of OCT results at this point of time. These issues must be resolved before OCT can be accepted for widespread clinical use in glaucoma. Apart from this note of caution the potential utility of OCT as a glaucoma diagnostic tool is extremely high as, adequate data exist to evaluate the patients in conjunction with other clinical parameters. A patient can be followed over time, using his or her own baseline. The two eyes of the patient can be compared for asymmetry, and a single eye can be examined for focal or sectoral NFL thinning.

All pieces of the glaucoma puzzle must be put together in order to care appropriately for the patient. The clinician must correlate clinically with IOP, ONH and NFL appearance, visual field data, as well as quantitative data contributed by technology, to detect glaucoma and its progression.

\section{REFERENCES}

1. Rand AR. Shield's textbook of glaucoma. (6th edn). Lippincott: Williams and Wilkins; 2011;77-78.

2. Robert LS, Mark FL, Michael VD. Becker-Shaffer's diagnosis and therapy of the glaucomas. (8th edn). Mosby: Elsevier; 2009;175-81.

3. Sommer A, Katz J, Quigley HA, et al. Clinically detectable nerve layer atrophy precedes the onset of glaucomatous field loss. Arch Ophthalmol 1991;109:77-83.

4. Tarek MS, Mark BS, Roger AH, Jonathan GC. Glaucomamedical diagnosis and therapy. Saunders: Elsevier; 2009;1: 232-35.

5. Schuman JS, Hee MR, Fuliafito CA, et al. Quantification of nerve fibre layer thickness in normal and glaucomatous eyes using optical coherence tomography. Arch Ophthalmol 1995;113:586-96.

6. Leung CK, Cheung CY. Weinreb RN, et al. RNFL imaging with Spectral-domain OCT: A variability and diagnostic performance study. Ophthalmology 2009;116:1257-63.

7. Leung CK, Chan WM, Yung WH, et al. Comparison of macular and peripapillary measurements for the detection of glaucoma: An OCT study. Ophthalmology 2005;112:391-400.

8. Parikh RS, Parikh S, Sekhar GC, et al. Diagnostic capability of OCT (Stratus OCT 3) in early glaucoma. Ophthalmology 2007;114:2238-43.

9. Chang RT, et al. Sensitivity and specificity of Time-domain versus Spectral-domain OCT in diagnosing early to moderate glaucoma. Ophthalmology 2009;116:2294-99. 